\title{
Evaluation of the Role of Routine Laboratory Biomarkers in COVID-19 Patients: Perspective from a Tertiary Care Hospital in India
}

\author{
Barnali Das $^{1} \cdot$ Seema Y. Bhatia ${ }^{1}$ Poonam M. Pal ${ }^{1}$
}

Received: 22 August 2020/ Accepted: 17 February 2021 / Published online: 23 April 2021

(C) Association of Clinical Biochemists of India 2021

\begin{abstract}
To evaluate the role of routine laboratory biomarkers like C Reactive Protein (CRP), Lactate Dehydrogenase (LDH), Interleukin 6 (IL6), Ferritin, Creatinine, Procalcitonin (PCT), Aspartate aminotransferase (AST), Alanine aminotransferase (ALT), Serum Albumin, Total Bilirubin (T Bil), High Sensitive Troponin I (hs troponin I), N Terminal-pro B-type Natriuretic Peptide (NT proBNP), Blood Urea Nitrogen (BUN) and Blood Gases in COVID 19 patients who are admitted with SARS CoV-2 positive test results by real-time reverse transcriptase polymerase chain reaction (rRT PCR) in Kokilaben Dhirubhai Ambani Hospital \& Medical Research Institute, Mumbai, India. 100 individuals detected with COVID-19 belonging to the age group 12-83 years (median age 62 years) within the period of 1st March 2020 to 10th July 2020 were studied. The case group consisted of 72 males and 28 females. 40 healthy adults without any history or clinical evidence suggestive of COVID-19 and without any comorbidities, like diabetes, hypertension chronic lung disease, cardiac disease, cancer, and immune-compromised individuals were considered as a control group for the study. Routine laboratory findings of these 100 patients were used to evaluate the abnormalities found in COVID-19 patients. Statistical analysis was carried out on the data after determining whether the data had a normal/log-normal distribution and their significance was determined by calculating the p-value. The percentage of patients showing a decrease or increase from the normal value was calculated. Trend analysis was carried out for the
\end{abstract}

Barnali Das

drbarnalid@gmail.com

1 Department of Biochemistry \& Immunology, Kokilaben Dhirubhai Ambani Hospital and Medical Research Institute, Mumbai, Maharashtra, India
100 patients considered in the case group. Among them, 6 patients were used as representatives to show the trend in these biomarkers during the course of hospital stay. These 5 severe cases consisted of 2 adult males, 2 adult females, and 1 adolescent girl. This selection is to demonstrate the representation of COVID-19 infection in adult males and females and pediatric multisystem inflammatory syndrome associated with COVID-19 in the younger age group. One mild case (adult male) was also selected in the case study. We found a significant increase in mean values of AST, ALT, Total Billirubin, Creatinine, CRP, PCT, LDH, IL6, Ferritin, Lactate, hsTroponin I, NT Pro BNP and decrease in mean values of Albumin, SO2, and PO2 in COVID 19 cases than control. We applied Receiver Operating Curve (ROC) curve to discriminate case population more precisely than the control population. Therefore, Routine laboratory biomarkers appear to play a significant role in COVID-19 patients.

Keywords SARS-CoV-2 - COVID 19 - ARDS ·

Laboratory medicine $\cdot$ Biomarkers

\section{Introduction \& Background}

Coronavirus disease 2019 (COVID-19), is caused by a virus that belongs to the family of Coronaviridae [1]. It is a form of respiratory and systemic zoonosis that was originated in Wuhan (China) and is now a pandemic that has spread worldwide [1,2]. The virus is a novel enveloped RNA beta-coronavirus known as severe acute respiratory syndrome coronavirus 2 (SARS-CoV-2) [3, 4]. The SARSCoV-2 causing the coronavirus disease 2019 (COVID-19) has a large clinical spread that included patients with asymptomatic infection, severe viral pneumonia with 
respiratory tract failure, or mild upper respiratory tract infection [2]. COVID-19 has been reported as a public health concern as per World Health Organization (WHO) [3]. According to the World Health Organization (WHO), there have been 140,322,903 confirmed cases, with 3,003,794 deaths, as of 18th April, 2021 [5].

The virus mainly transmits through respiratory droplets, direct human to human contact, or by touching the nose, eyes, or mouth after touching a contaminated surface $[3,6,7]$. As per Centre of Disease Control (CDC), people with COVID-19 have had a wide range of symptoms reported-ranging from mild to severe illness. The incubation period for the same is $2-14$ days after exposure to the virus.

Further, as per CDC, major symptoms of COVID-19 include fever, chills, cough, tiredness, muscle or body aches and pains, and sore throat. In some other cases, congestion or runny nose, nausea or vomiting, diarrhea, skin manifestations (rash) headache, and loss of taste or smell are also observed [3]. Other serious symptoms or visible emergency warning signs are difficulty in breathing or shortness of breath, persistent chest pain or pressure in the chest, new confusion, inability to wake or stay awake, bluish marks on lips or face, and dyspnea. [2, 6, 8, 9]. ARDS of unknown origin, fever, and fatigue were the most common symptoms seen in patients initially in December 2019 in Wuhan [9].

The SARS-CoV-2 has an effect on the respiratory system leading to the rapid development of pneumonia $[2,6,8]$. It also leads to acute respiratory distress syndrome (ARDS) and multi-organ failure in case of severe infection which might lead to death. It is seen that $10-15 \%$ of the COVID-19 cases may lead to severe interstitial pneumonia that may progress to ARDS [10]. The risk of severity and mortality has been observed to be much higher in older people and in patients with underlying comorbidities, such as hypertension, diabetes, cardiac disease, chronic lung disease, cancer, and immune-compromised individuals $[2,11-13]$.

Unlike the previous coronavirus diseases, i.e. Middle East respiratory syndrome (MERS) and Severe acute respiratory syndrome (SARS), COVID-19 has relatively low pathogenicity and longer incubation. However, this SARS $\mathrm{CoV}-2$ virus is highly contagious [1].

In case of children, very few showed severe symptoms. It was observed that children were mostly asymptomatic COVID-19 positive [14]. However, there have been few reports involving children and adolescents in intensive care units having Kawasaki disease-like symptoms, with case reports of hyper-inflammatory syndrome, multi-organ failure, and shock [15-17]. This syndrome is named Pediatric Inflammatory Multisystem Syndrome temporally associated with SARS-COV-2 (PIMS-TS). PIMS-TS appear to affect older children, gastrointestinal symptoms (particularly abdominal pain) predominate and there appears to be a greater chance of damage to the heart and kidneys [15-17].

The critical role of laboratory medicine in this pandemic extends far more than the etiological diagnosis of COVID19. In spite of the broadly defined clinical characteristics of COVID-19, we still lack the understanding of the abnormalities in the laboratory findings in COVID-19 patients. Since laboratory medicine plays an important role in the early detection, diagnosis, prognosis as well as management of the disease we can use this tool for a better understanding of this novel coronavirus disease [18]. Also, the lack of specific treatment towards this disease [6], early diagnosis becomes a very important factor. Biochemical monitoring of COVID-19 patients through testing is critical for assessing disease severity and progression, as well as monitoring of therapeutic intervention [1]. In addition to more common laboratory tests like Liver Function Test (LFT), Kidney Function Test (KFT), Blood Gas, etc., evidence suggests that patients with severe COVID-19 could be at risk of cytokine storm syndrome [1-3, 5-8, 10-12]. Cytokine tests, particularly IL-6, should be used to assess patients exhibiting severe illness and those suspected of hyper-inflammation [10, 18, 19]. Such patients with features of systemic hyper-inflammation are categorized display macrophage activation syndrome (MAS) or cytokine storm [18].

Few studies have been published globally to investigate the role of biochemical markers \& immunoassay markers in COVID 19 patients $[1-3,5-7,10]$.

The essential role of Biochemistry, Immunology, and routine pathology laboratory is well known in the modern healthcare system.

These Laboratory biomarkers play an essential role in the patient admission protocol, assessment of staging of disease according to severity, prognostication, patient monitoring, and therapeutic guide [18-21].

In this study, we have investigated the routine biochemistry and immunology laboratory findings of the patients infected with SARS-CoV-2. The laboratory biochemical parameters considered include Creatinine, Albumin, Interleukin-6, Ferritin, Aspartate aminotransferase (AST), Alanine aminotransferase (ALT), Lactate dehydrogenase (LDH), C-reactive protein (CRP), hs Troponin I, Total Bilirubin, Blood Urea Nitrogen (BUN), NT-proBNP, Procalcitonin (PCT) and Blood gas levels. We have analysed the above biomarkers with an aim to determine the significant trend analysis shown by COVID-19 positive patients in their blood reports hoping for better identification and monitoring of the disease. 


\section{Materials and Methods}

We have carried out a retrospective study involving COVID-19 patients admitted to Kokilaben Dhirubhai Ambani Hospital \& Medical Research Center, Mumbai. A total of 100 patients with confirmed COVID-19 disease (case group) belonging to the age group of 12-83 years (median age 62 years) within the period of 1st March 2020 to 10th July 2020 was considered for the study. 40 healthy adults without any history and clinical evidence suggestive of COVID-19 and without any comorbidities, like diabetes, hypertension chronic lung disease, cardiac disease, cancer, and immuno-compromised individuals were considered as a control group for the study. The case group consists of 72 males with an age range of 29-85 years (median age 63 years) and 28 females with an age range of $12-85$ years (median age of 56 years). There are two adolescent girls included in the case group. These patients belonging to the case group were confirmed positive for SARS CoV-2 by collecting nasopharyngeal swab specimens and subjecting them to real-time reverse transcriptase polymerase chain reaction (rRT-PCR) for SARS-CoV2 virus. Among the 100 patients that belong to the case group, 20 patients (median age 57 years) were found to have mild COVID-19 infection. This mild COVID-19 patient group consisted of 10 males with an age range of 38-81 years (median age 57 years) and 10 females with an age range of $42-85$ years (median age 57 years).

We have performed a trend analysis of 100 patients (case group) for disease monitoring purpose, from which we'll here refer severe cases of 2 adult males, 2 adult females, and 1 adolescent girl as representative example to show the laboratory biomarker trend analysis in COVID-19 patients admitted in the Intensive Care Unit with a probable diagnosis of COVID-19 with ARDS and cytokine storm. One mild case (adult male) was also selected in the case study.

Laboratory parameters tested during the course of admission and treatment for COVID-19 were displayed individually to determine the change in their levels (daywise).

The data for laboratory biomarkers considered here were collected from the routine blood test carried out from the COVID-19 patients sample on admission. IL-6, PCT, NT Pro BNP was analysed on Roche COBAS e411 (Roche Diagnostics, Basel, Switzerland). CRP, AST, ALT, GGT, Total Bilirubin, Albumin, LDH, Creatinine, BUN were measured using Roche COBAS 6000 (Roche Diagnostics, Basel, Switzerland). Ferritin and hsTroponin I was measured using either ARCHITECT i1000sr or ARCHITECT i2000sr (Abbott Diagnostics, Abbott Park, IL, USA). Blood gas levels were estimated using RAPID LAB 1265 System (Siemens Healthcare Private Limited, India).

\section{Statistical Analysis}

The data were analyzed using Minitab (Minitab Inc, PA) and Prism (Graph pad Software, San Diego, CA) statistical software package. Before carrying out the statistical analysis, the normal and log-normal distribution of the data was confirmed. To determine the normal distribution of the data, a histogram with a fit curve was used and a probability plot was also used for the confirmation of the same. The significance of the data to differentiate case from control group having normal / log-normal distribution was determined by performing a t-test. For the data to be significant the $p$-value has to be $<0.05$. Receiver Operating Curve (ROC) analysis was carried out for all the parameters considered in the study to discriminate case population more precisely than the control population. The patient data was then used to calculate the percentage of patients showing an increase or decrease in the parameters. The study of the trend analysis shown by the individual parameter during the course of the hospital stay was carried out by plotting a smooth curve scatter plot for routine laboratory parameters in the patients of COVID-19 disease considered in the case group.

\section{Results}

The data obtained from the laboratory findings were checked for its distribution by plotting a histogram with a fit curve. For example, Fig. 1 shows Ferritin values do not follow a normal distribution in the histogram. Therefore, to confirm, we plotted the probability plot to confirm. The probability plot indicates that the data considered for the study lies within $95 \%$ confidence interval of log-normal fit.

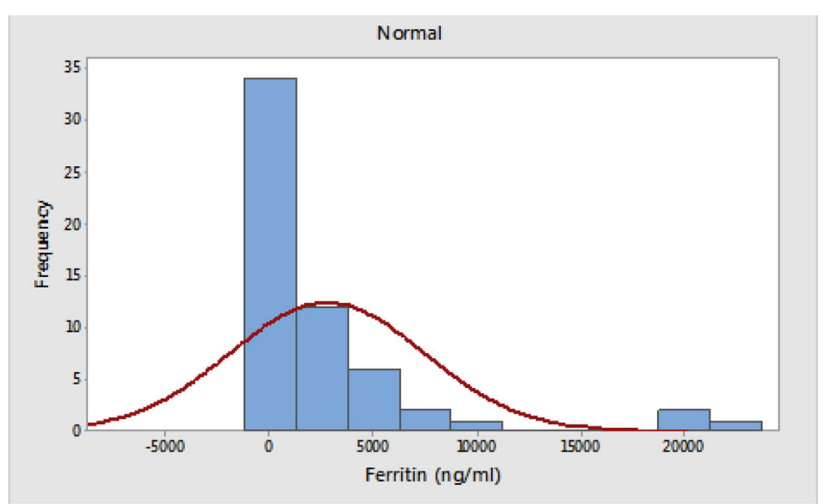

Fig. 1 Histogram of Ferritin 
All the parameters were checked for the probability distribution. The histogram with a fit curve (Fig. 1) and probability plot (Fig. 2) of Ferritin is shown below as representative.

$T$-test was carried out and p-value was calculated to know if the data is significant to differentiate between the case and control for further statistical analysis.

After calculating the p-value, the abnormalities shown in the blood reports of COVID-19 positive patients were studied by calculating the percentage of patients showing an increase or decrease in a particular analyte.

Receiver Operating Curve (ROC) analysis was carried out for all the parameters. The area under the curve for Ferritin was found to be 0.9121 with $95 \%$ confidence interval $(\mathrm{CI})$ and a $p$-value $<0.0001$. The area under the curve for IL- 6 was found to be 1.0 with $95 \%$ confidence interval $(\mathrm{CI})$ and a $p$-value $<0.0001$. The area under the curve for $\mathrm{PO} 2$ was found to be 0.7866 with $95 \%$ confidence interval (CI) and a $p$-value $<0.0001$. The area under the curve for SO2 was found to be 0.7170 with $95 \%$ confidence interval $(\mathrm{CI})$ and a $p$-value $<0.0001$. The area under the curve for CRP was found to be 0.9639 with $95 \%$ confidence interval (CI) and a $p$-value $<0.0001$. The area under the curve for PCT was found to be 0.7911 with $95 \%$ confidence interval (CI) and a $p$-value $<0.0001$. The area under the curve for hs Troponin I was found to be 0.8543 with $95 \%$ confidence interval $(\mathrm{CI})$ and a $p$-value $<0.0001$. The area under the curve for NT proBNP was found to be 0.9815 with $95 \%$ confidence interval (CI) and a $p$ value $<0.0001$. The area under the curve for LDH was found to be 0.9813 with $95 \%$ confidence interval (CI) and a $\mathrm{p}$-value $<0.0001$. The area under the curve for Creatinine was found to be 0.8536 with $95 \%$ confidence interval (CI) and a $p$-value $<0.0001$. The area under the curve for BUN was found to be 0.7170 with $95 \%$ confidence interval (CI) and a $p$-value $<0.0001$. The area under the curve for AST

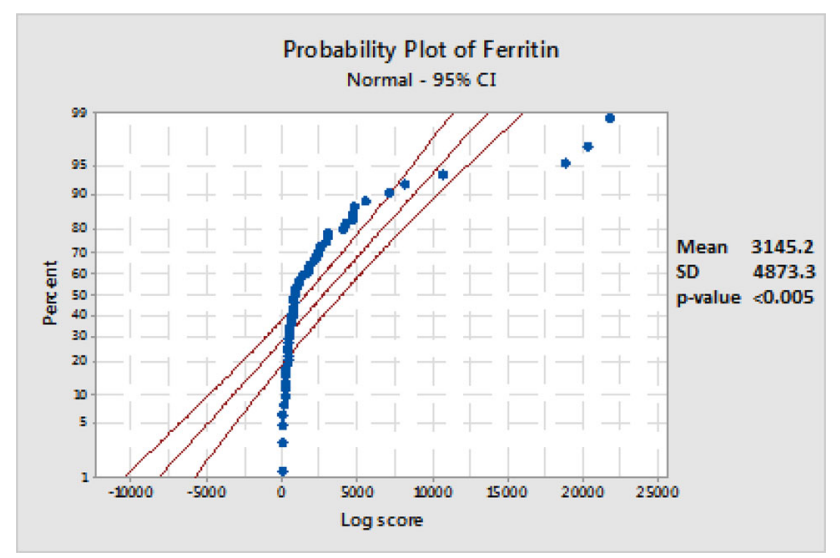

Fig. 2 Probability plot of Ferritin showing fit to Log-Normal distribution was found to be 0.8845 with $95 \%$ confidence interval (CI) and a $p$-value $<0.0001$. The area under the curve for ALT was found to be 0.8112 with $95 \%$ confidence interval (CI) and a $p$-value $<0.0001$. The ROC curve of Ferritin is represented here as a reference (Fig. 3a).

Additionally ROC analysis was also carried out on the mild positive group (20 patients). The area under the curve for Ferritin was found to 0.7125 with $95 \%$ confidence interval $(\mathrm{CI})$ and a $p$-value $<0.0001$. The area under the curve for IL- 6 was found to be 1.0 with $95 \%$ confidence interval $(\mathrm{CI})$ and a $p$-value $<0.0001$. The area under the curve for PO2 was found to be 0.687 with $95 \%$ confidence interval $(\mathrm{CI})$ and a $p$-value $<0.0001$. The area under the curve for $\mathrm{SO} 2$ was found to be 0.6625 with $95 \%$ confidence interval $(\mathrm{CI})$ and a $p$-value $<0.0001$. The area under the curve for CRP was found to be 0.9683 with $95 \%$ confidence interval $(\mathrm{CI})$ and a $p$-value $<0.0001$. The area under the curve for PCT was found to be 0.7156 with $95 \%$ confidence interval (CI) and a $p$-value $<0.0001$. The area under the curve for hs Troponin I was found to be 0.7063 with $95 \%$ confidence interval (CI) and a $p$-value $<0.0001$. The area under the curve for NT proBNP was found to be 1.0 with $95 \%$ confidence interval $(\mathrm{CI})$ and a $p$-value $<$ 0.0001 . The area under the curve for LDH was found to be 1.0 with $95 \%$ confidence interval $(\mathrm{CI})$ and a $p$-value $<$
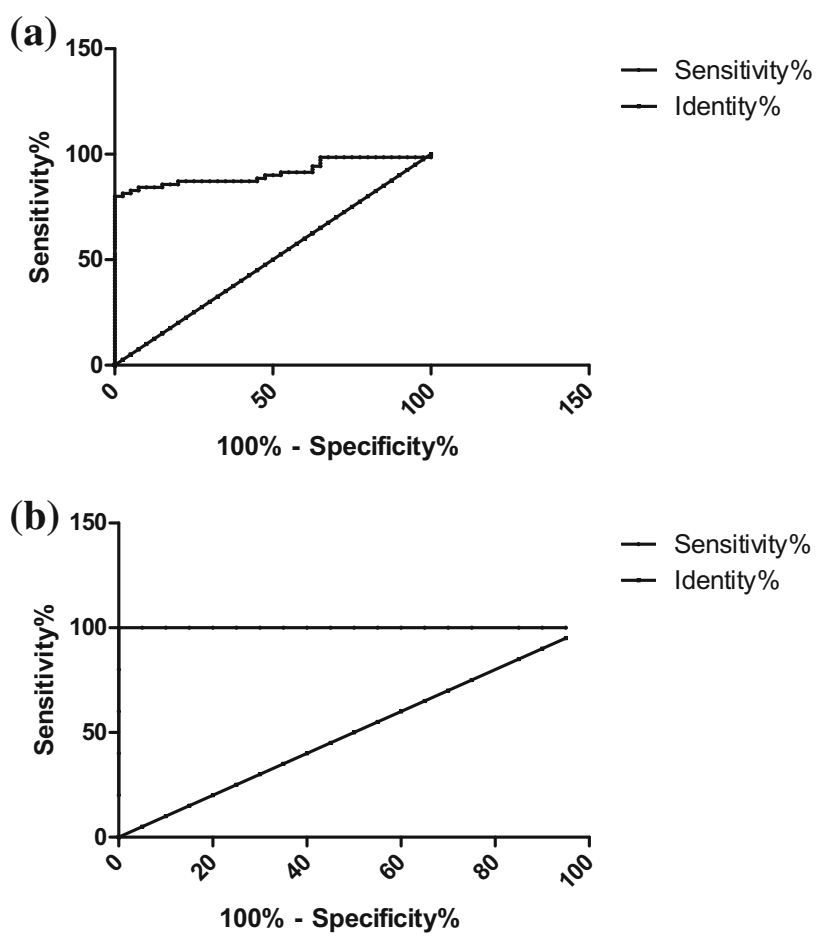

Fig. 3 a Receiver Operating Curve (ROC) curve for Ferritin. b Receiver Operating Curve (ROC) curve for IL-6. The Area under curve (AUC) depicts the following: AUC 0.90-1: excellent accuracy, $0.80-0.90$ : good accuracy, $0.70-0.80$ : fairly accurate, $0.60-0.70$ : poor accuracy; $0.50-0.60$ : failure 
0.0001 . The area under the curve for Creatinine was found to be 0.7195 with $95 \%$ confidence interval $(\mathrm{CI})$ and a $p$ value $<0.0001$. The area under the curve for BUN was found to be 0.5204 with $95 \%$ confidence interval (CI) and a $p$-value $<0.0001$. The area under the curve for AST was found to be 0.8250 with $95 \%$ confidence interval (CI) and a $p$-value $<0.0001$. The area under the curve for ALT was found to be 0.7616 with $95 \%$ confidence interval (CI) and a $p$-value $<0.0001$. The ROC curve of IL-6 in the mild COVID-19 positive patient group is represented here as a reference (Fig. 3b).

We analyzed the retrospective routine laboratory data from 1st March 2020 to 10th July 2020 of the COVID 19 cases admitted in Kokilaben Dhirubhai Ambani Hospital \& Medical Research Institute.

Amongst the patients belonging to the case group, $73 \%$ of the patients showed elevated IL-6 levels, 56\% of patients show an increase in Ferritin levels, 79\% of the patients show an elevation in CRP levels, $80 \%$ of the patients show elevated levels of D-dimer, 30\% show elevation in PCT levels, $25 \%$ of the patients show an elevation in hs Troponin I and $26 \%$ of the patients show an elevation in NT proBNP levels, $42 \%$ of patients show elevated LDH levels, $31 \%$ of patients show elevated creatinine levels, $36 \%$ of the patients show elevated BUN levels, $40 \%$ of patients show elevated AST levels and $23 \%$ of patients show elevated ALT levels. On the other hand, 58\% show a decrease in PO2 levels, 52\% show a decrease in SO2 levels and $56 \%$ of patients show decreased levels of Albumin.

Table 1 Percentage of patients showing Increase $(\uparrow) /$ Decrease $(\downarrow)$ in the biomarkers:

\begin{tabular}{ll}
\hline Parameters & $\begin{array}{l}\text { Percentage of abnormal results in } \\
\text { total no. of patients (case group) }\end{array}$ \\
\hline IL-6 & $73 \uparrow$ \\
PO2 & $58 \downarrow$ \\
SO2 & $52 \downarrow$ \\
CRP & $79 \uparrow$ \\
PCT & $30 \uparrow$ \\
hsTroponin I & $25 \uparrow$ \\
NtProBNP & $26 \uparrow$ \\
AST & $40 \uparrow$ \\
ALT & $23 \uparrow$ \\
Albumin & $56 \downarrow$ \\
D-dimer & $80 \uparrow$ \\
Ferritin & $56 \uparrow$ \\
LDH & $42 \uparrow$ \\
Creatinine & $31 \uparrow$ \\
BUN & $36 \uparrow$ \\
\hline
\end{tabular}

$N=100$
Table 1 summarizes the percentage of patients showing abnormal results of the parameters considered in the study population.

When biomarkers were compared with hematological markers, lymphopenia was observed in $70 \%$ of the patients, and neutrophilia was observed in $61 \%$ of the patients. Also, a quantitative estimation of the abnormalities seen in COVID-19 case group was calculated. Table 2 shows the abnormalities in the parameters considered in COVID-19 case group.

To explain the role of routine laboratory parameters in disease monitoring, we have performed a trend analysis of 100 patients amongst which we have selected two adult males, two adult females, and one adolescent girl as representative examples to show the laboratory biomarker trend analysis for monitoring in COVID 19 patients (severe infection). This selection is to demonstrate the representation of COVID-19 infection in adult male and female and pediatric multisystem inflammatory syndrome associated with COVID-19 in the younger age group. Additionally, a mild COVID-19 positive case (adult male) was also selected.

In this assessment, the daily laboratory finding of the patients was used to plot a smooth curve scatter plot and see the fluctuation in the parameters during the course of COVID-19 treatment. We are showing the figures of trend analysis of the following selected biomarkers as representative parameters: CRP, Ferritin, LDH, IL6, PCT, hs Troponin I, NT Pro BNP, SO\%, Lactate, Creatinine, BUN, ALT and AST.

\section{Case 1}

An adult male, who was a known case of diabetes was presented to the hospital with a complaint of fever for 2 days, along with breathing difficulty and generalized weakness for 5 days. The patient had a12 days travel history. The patient tested positive for SARS-CoV-2 by reverse transcriptase polymerase chain reaction (rRT-PCR) using a nasopharyngeal swab. Chest X-ray showed bilateral lung infiltrates. Routine laboratory findings supported the cytokine storm with elevated levels of Interleukin-6 (204 pg/ml), C Reactive Protein (20.77 ng/ml), Procalcitonin $(1.808 \mathrm{ng} / \mathrm{ml})$, High Sensitive Troponin I (49.29 pg/ $\mathrm{ml})$, NT proBNP (232.2 pg/ml), D-dimer (1364.47 ng/ml), Ferritin (718.7 ng/ml), LDH (921.3 U/L), Blood Urea Nitrogen (31.2 mg/dl), AST (223.3 U/L), ALT (194.5 $\mathrm{U} / \mathrm{L})$, Lactate $(3.5 \mathrm{mmol} / \mathrm{L}), \mathrm{WBC}\left(15.5 \times 10^{3} / \mathrm{microL}\right)$, Neutrophil count (94.2\%), Absolute Neutrophil count $\left(14.7 \times 10^{3} / \mathrm{microL}\right)$; and decreased levels of Albumin $(2.6 \mathrm{~g} / \mathrm{dl})$, Total Protein $(5.8 \mathrm{~g} / \mathrm{dl})$, Lymphocyte count (3.9\%), and Absolute Lymphocyte count (0.6 X 10\% $\frac{3}{\mathrm{mi}-}$ croL), $\mathrm{SO} 2 \%(78.4 \%)$. 
Table 2 Quantitative representation of abnormalities in COVID-19 patients admitted in Intensive care Unit

\begin{tabular}{lcc}
\hline Parameter & \multicolumn{1}{l}{ Mean } & \multicolumn{1}{c}{ Mean \pm SD } \\
\hline IL-6 & 1043.316 & $127 \pm 6015.724$ \\
PO2 & 52.30769 & $48.95 \pm 18.73178$ \\
SO2 & 74.63043 & $81.75 \pm 19.73366$ \\
CRP & 12.79094 & $8.107 \pm 12.64244$ \\
PCT & 33.72115 & $2.245 \pm 97.19951$ \\
hsTroponin I & 7928.504 & $239.35 \pm 21,655.5294$ \\
NtProBNP & $12,770.39$ & $4739 \pm 17,549.09$ \\
AST & 338.2786 & $75.65 \pm 1264.352$ \\
ALT & 313.7842 & $69 \pm 1052.178$ \\
Albumin & 0.4458 & $0.285 \pm 0.599643$ \\
D Dimer & $10,692.7$ & $1668.12 \pm 46,147.59$ \\
Ferritin & 3145.221 & $1141.83 \pm 4873.384$ \\
LDH & 601.635 & $415.35 \pm 1055.807$ \\
Creatinine & 12.70964 & $2.345 \pm 36.29011$ \\
BUN & 41.5875 & $36.9 \pm 19.95802$ \\
\hline
\end{tabular}

$N=100$

\section{Case 2}

An adult male who was a known case of diabetes, hypertension, and ischemic heart disease (with a past history of stroke 7 months ago) was presented to the hospital with a complaint of headache, followed by giddiness and stiffness of upper limb, frothing from mouth and up-rolling of eyes followed by unresponsiveness. The patient had no travel history. The patient developed high-grade fever spikes with raised infective markers. The patient tested positive for SARS-CoV-2 by reverse transcriptase polymerase chain reaction (rRT-PCR) using a nasopharyngeal swab. The routine laboratory findings depicted cytokine storm with elevated levels of Interleukin-6 (2766 pg/ml), C Reactive Protein $(29.72 \mathrm{ng} / \mathrm{ml})$, Procalcitonin $(17.18 \mathrm{ng} / \mathrm{ml})$,Ddimer $(6646.5 \mathrm{ng} / \mathrm{ml})$, Ferritin $(5827.69 \mathrm{ng} / \mathrm{ml})$, LDH (291.8 U/L), Creatinine (1.12 mg/dl), Blood Urea Nitrogen $(28.5 \mathrm{mg} / \mathrm{dl})$, Lactate $(2.8 \mathrm{mmol} / \mathrm{L})$, WBC $\left(13.5 \times 10^{3} /\right.$ microL), Neutrophil count (91\%), Absolute Neutrophil count $\left(12.63 \times 10^{3} / \mathrm{microL}\right)$; and decreased levels of Lymphocyte count (5\%), Absolute Lymphocyte count $\left(0.74 \times 10^{3} / \mathrm{microL}\right), \mathrm{SO} 2 \%(90.9 \%)$.

\section{Case 3}

An adolescent female, who was a known case of persistent oliguria, trachycardia, hypotension was presented to the hospital with a complaint of fever for 3 days, along with rash for 2 days. The patient had no travel history. The patient tested positive for SARS-CoV-2 by reverse transcriptase polymerase chain reaction (rRT-PCR) using a nasopharyngeal swab. Routine laboratory findings supported the diagnosis of PIMS-TS COVID-19 [14-16] with elevated levels of Interleukin-6 (272.2 pg/ml), C Reactive Protein $(22.12 \mathrm{ng} / \mathrm{ml})$, High Sensitive Troponin I (225.3 pg/ml), D-dimer ( $2479.4 \mathrm{ng} / \mathrm{ml})$,Lactate $(4.2 \mathrm{mmol} /$ L), Ferritin $(401.5 \mathrm{ng} / \mathrm{ml})$, WBC $\left(12.1 \times 10^{3} / \mathrm{microL}\right)$, Neutrophil count (90.4\%),Absolute Neutrophil count $\left(10.94 \times 10^{3} / \mathrm{microL}\right)$; and decreased levels of Lymphocyte count $(5.7 \%)$, Absolute Lymphocyte count $\left(0.69 \times 10^{3} /\right.$ microL), Albumin (2.78 g/dl), Total Protein $(5.75 \mathrm{~g} / \mathrm{dl})$.

\section{Case 4}

An adult female patient was presented to the hospital with a complaint of fever and loose stools. The patient had no travel history. The patient tested positive for SARS-CoV-2 by reverse transcriptase polymerase chain reaction (rRTPCR) using a nasopharyngeal swab. The routine laboratory findings elevated levels of Interleukin-6 $(25.35 \mathrm{pg} / \mathrm{ml}), \mathrm{C}$ Reactive Protein $(3.38 \mathrm{ng} / \mathrm{ml})$, D-dimer $(976.73 \mathrm{ng} / \mathrm{ml})$, Ferritin (489.9 ng/ml), Creatinine $(15.2 \mathrm{mg} / \mathrm{dl})$; and decreased levels of Lymphocyte count (11.0\%), Absolute Lymphocyte count $\left(0.97 \times 10^{3} / \mathrm{microL}\right)$, Albumin $(3.84 \mathrm{~g} /$ $\mathrm{dl}), \mathrm{SO} 2 \%(81.5 \%)$.

The trend analysis of case 1 to case 4 is shown in Fig. 4a-m.

\section{Case 5}

An adult female who is a known case of Hypothyroidisum presented to the hospital with a complaint of fever and breathlessness for 3-4 days along with a history of unrolling of eyeball, stiffness of upper limb, frothing from the mouth. The patient had no travel or contact history. The patient was tested positive for SARS-CoV-2 by reverse transcriptase polymerase chain reaction (rRT-PCR) using a nasopharyngeal swab. High-Resolution Computed Tomography (HRCT) chest showed diffuse ground-glass opacities with interlobular septal thickness are seen scattered in both the lung field predominantly involving bilateral basal lobes. The routine laboratory findings elevated levels of Procalcitonin (396.44 ng/ml), Ferritin (21822 ng/ $\mathrm{ml})$, Creatinine $(129 \mathrm{mg} / \mathrm{dl})$, Blood Urea Nitrogen $(26.5 \mathrm{mg} / \mathrm{dl})$ and Lactate $(4.3 \mathrm{mmol} / \mathrm{L})$, WBC $\left(18.66 \times 10^{3} / \mathrm{microL}\right), \quad$ Absolute Neutrophil count $\left(14.4 \times 10^{3} / \mathrm{microL}\right)$ and decreased levels of Lymphocyte count (18.6\%), Absolute Lymphocyte count (3.46 X 103\% microL), Platelet count $\left(96 \times 10^{3} / \mathrm{microL}\right)$, Total Protein $(5.47 \mathrm{~g} / \mathrm{dl})$.

The trend analysis of case 5 is shown in Fig. 5a-c. Only 3 parameters were considered for trend analysis as the rest of the parameters had only a single value. 
(a) Trend analysis of IL-6 in Case 1, 2, 3, and 4

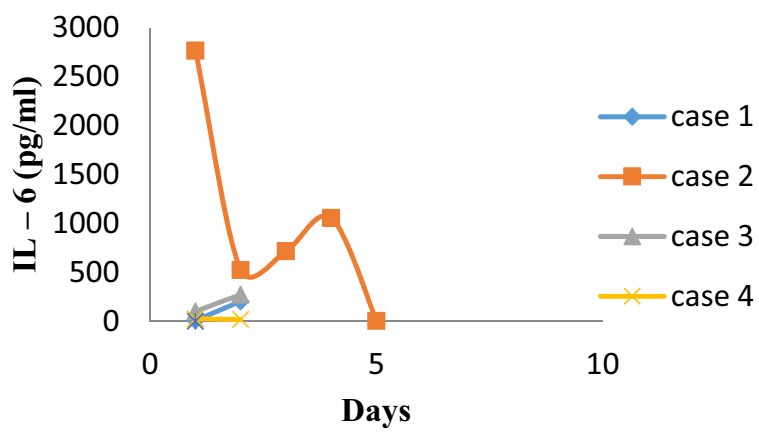

(b) Trend analysis of SO2 in Case 1, 2, 3, and 4

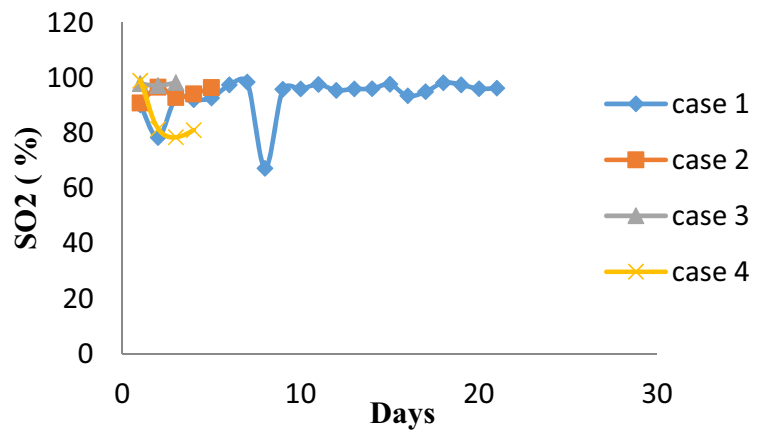

(c) Trend analysis of Lactate in Case 1, 2, 3, and 4

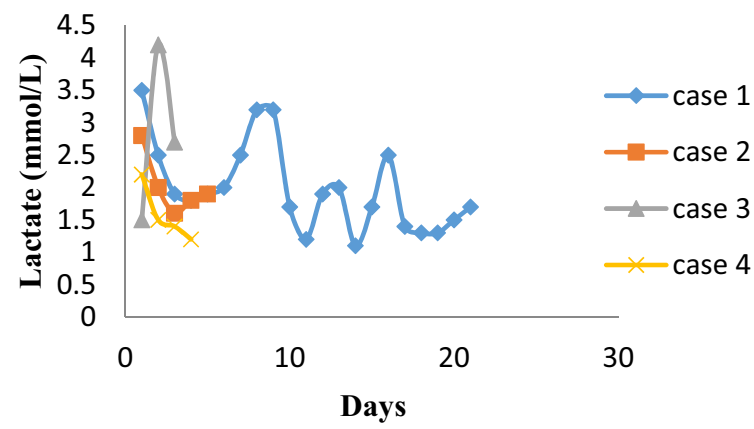

(d) Trend analysis of CRP in Case 1, 2, 3, and 4

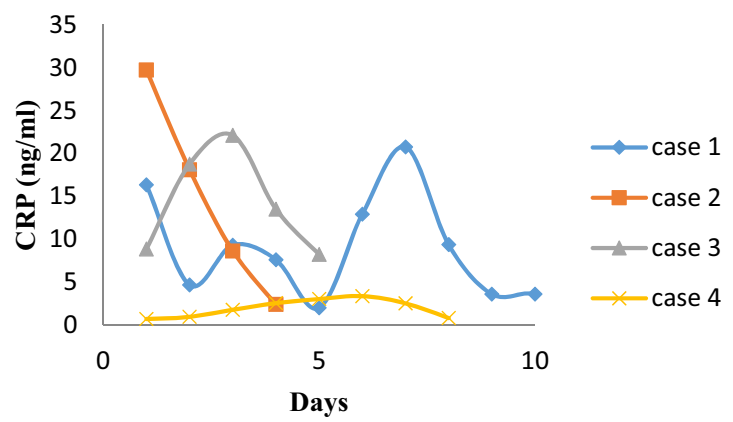

Fig. 4 Trend analysis of representative biomarkers in Case 1, 2, 3, and a Trend analysis of IL-6, b Trend analysis of SO2, c Trend analysis of Lactate, $\mathbf{d}$ Trend analysis of CRP, e Trend analysis of PCT, $\mathbf{f}$ Trend analysis of hs Troponin I, g Trend analysis of NT (e) Trend analysis of PCT in Case 1, 2, 3, and 4

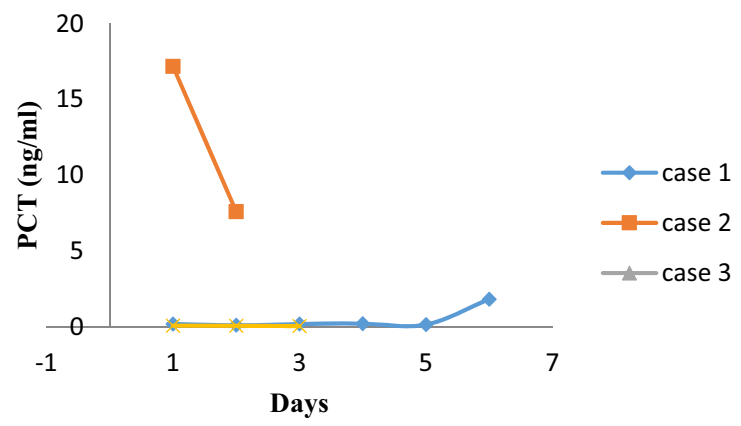

(f) Trend analysis of hs Troponin I in Case 1, 2, 3, and 4

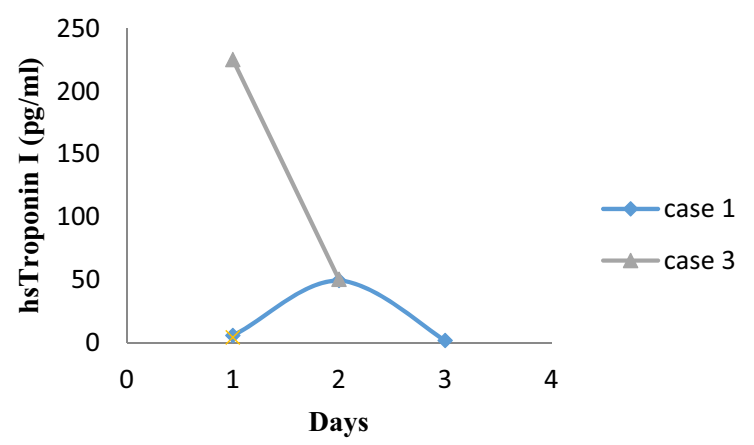

(g) Trend analysis of NT proBNP in Case 1, 2, 3, and 4

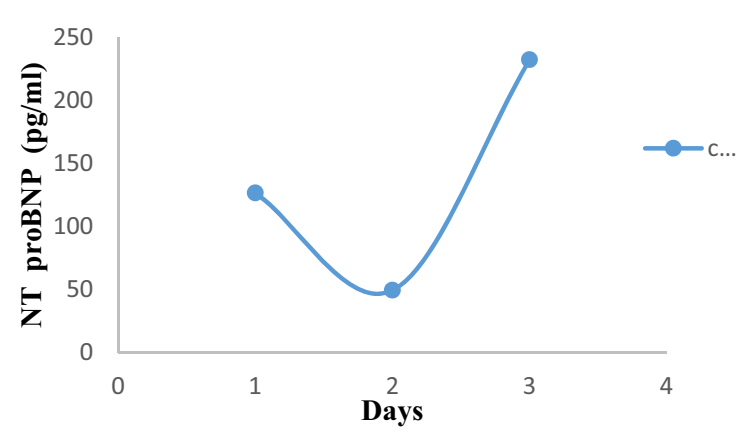

(h) Trend analysis of Ferritin in Case 1, 2, 3, and 4

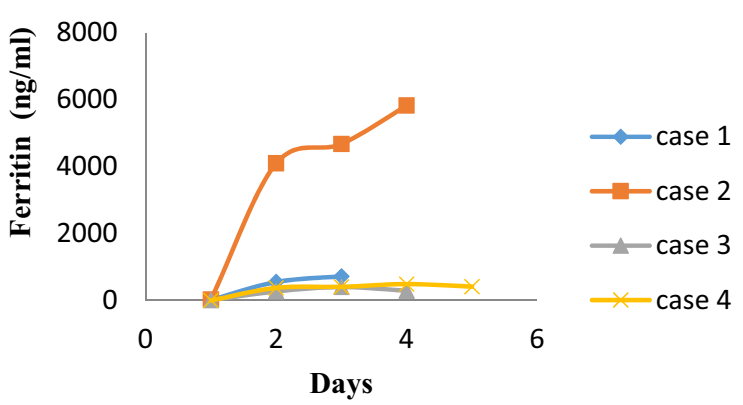

proBNP, $\mathbf{h}$ Trend analysis of Ferritin, i Trend analysis of LDH, $\mathbf{j}$ Trend analysis of Creatinine, $\mathbf{k}$ Trend analysis of BUN, $\mathbf{l}$ Trend analysis of ALT, $\mathbf{m}$ Trend analysis of AST 
(i) Trend analysis of $\mathrm{LDH}$ in Case 1, 2, 3, and 4

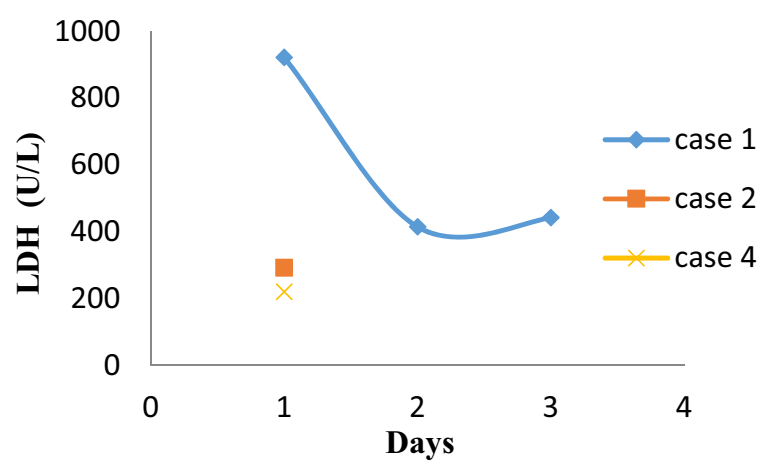

(j) Trend analysis of Creatinine in Case 1, 2, 3, and 4

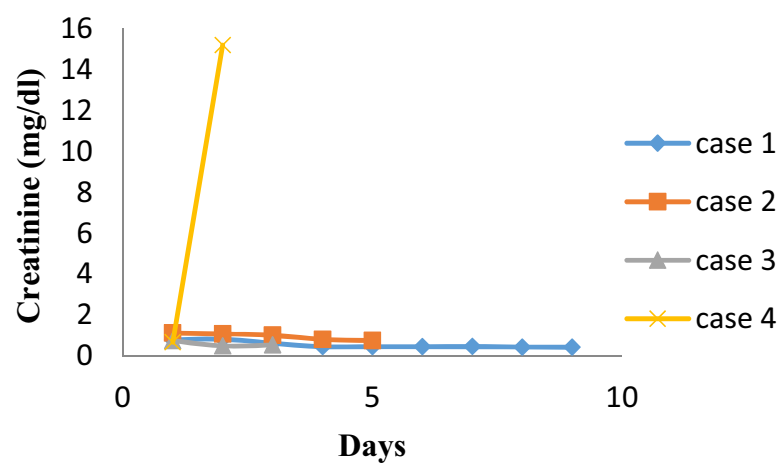

(k) Trend analysis of BUN in Case 1, 2,3, and 4

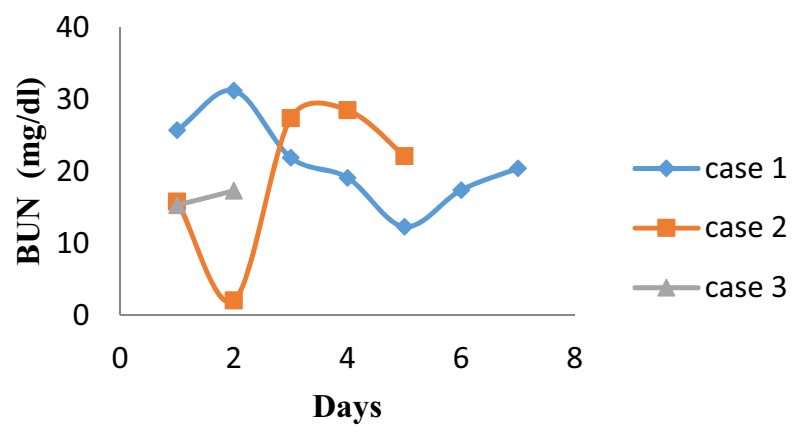

(l) Trend analysis of ALT in Case 1, 2, 3, and 4

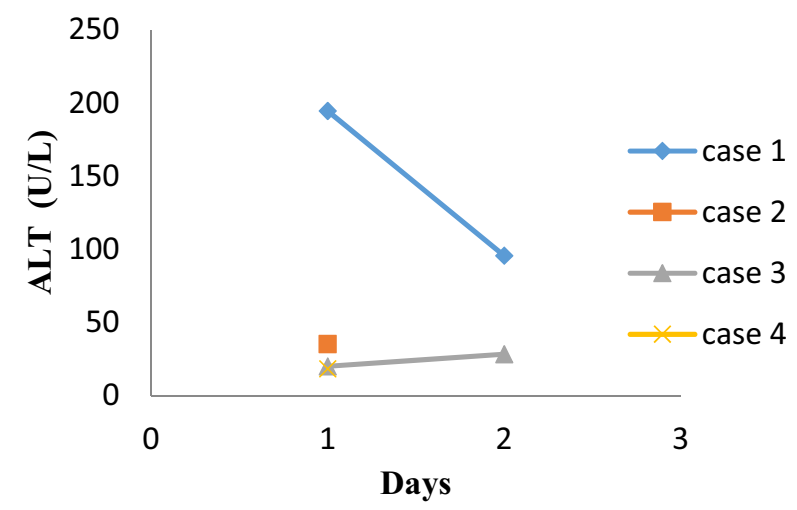

(m) Trend analysis of AST in Case 1, 2, 3, and 4

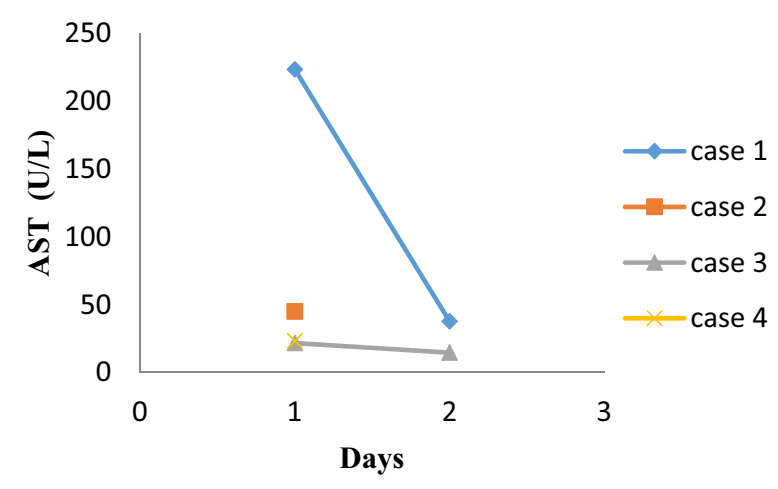

Fig. 4 continued

\section{Case 6}

A mild case of an adult male with complaints of dry cough from 3-4 days and low-grade fever since 4 days. The patient was tested positive for SARS-CoV-2by reverse transcriptase polymerase chain reaction (rRT-PCR) using a nasopharyngeal swab. The routine laboratory findings elevated levels of Interleukin-6 (49.32 pg/ml), C Reactive Protein $(6.318 \mathrm{ng} / \mathrm{ml})$, Ferritin $(476.69 \mathrm{ng} / \mathrm{ml})$, and decreased levels of Lymphocyte count (19.7\%), SO2\% $(93.9 \%)$. 
Here, we are giving examples of the importance of biomarker monitoring in admitted patients. For example, if we consider observing the trend of Interleukin- 6, C Reactive Protein, Procalcitonin, High Sensitive Troponin I, NT proBNP, Ferritin, LDH, Blood Urea Nitrogen, AST, ALT, Albumin, Total Protein, Lactate, SO2 and PO2 of one critical patient admitted in ICU over the course of hospital stay, we can see trend analysis of those biomarkers to chase cytokine storm. Table 3 shows the laboratory abnormalities observed in the COVID-19 progression.

\section{Discussion}

The ongoing pandemic of COVID-19 is characterized by respiratory illness and diverse systemic clinical presentations, which in turn are reflected by routine laboratory abnormalities, based on the severity of disease presentation [22]. The main laboratory changes encompass an array of increased inflammatory biomarkers, coagulation parameters, tissue-specific tissue injury indicators (liver, kidney, cardiac), and derangement of the complete blood count $[13,22-25]$. Based on the severity of the disease, the host inflammatory response to the virus may lead to a cytokine storm that can cause multi-organ damage [26]. Biomarkers of inflammation, cardiac and muscle injury as well as liver and kidney function and coagulation measures were also significantly deranged in patients with the critical stage of COVID-19 [27]. Interleukin 6 (IL-6), ferritin, CRP, PCT, D-dimer, LDH, etc. are indicators for the severe stage of disease [27].

In hospitalized patients, there should be close monitoring of biochemistry and immunoassay parameters like CRP, LFT, IL-6, and serum Ferritin, LDH, KFT, PCT, etc. as markers for potential progression to the critical stage of illness and fatality.

The entry of SARS CoV2 into the cell is mediated by the spike protein through the cell receptors binding, followed by the fusion of the membrane [28]. SARS-Cov-2 spike protein binds itself to angiotensin-converting enzyme 2 (ACE2) receptor. The host cell consists of type 2 transmembrane serine protease (TMPRSS2) that promotes viral uptake by cleaving ACE2 and activates the SARS-CoV-2 S protein. This process mediates the entry of coronavirus into alveolar epithelial type II pneumocytes. Alveolar epithelial type II cells show the expression of ACE2 and TMPRSS2. Multiplication of viral copy occurs inside the host cells. The infected cells and alveolar macrophage release inflammatory signal. Pulmonary edema with hyaline membrane formation leads to ARDS (acute respiratory distress syndrome) [29]. The above mechanism is the pathophysiology behind the laboratory biomarkers in COVID-19 patients. Once the virus enters the human body

\section{(a) Trend analysis of BUN in Case 5}

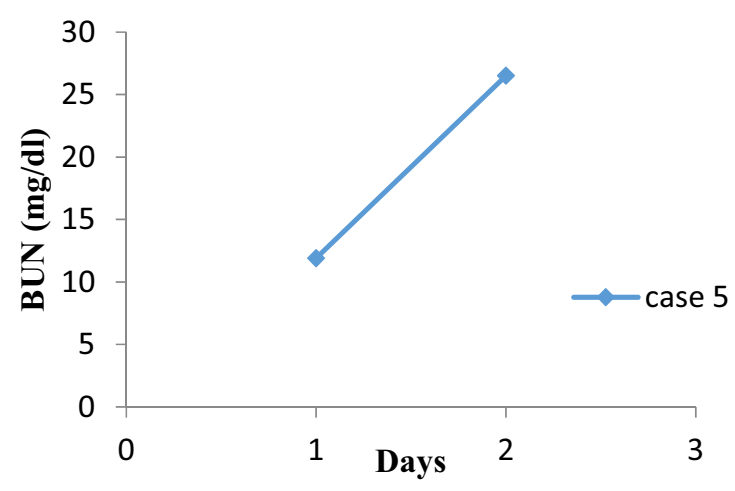

(b) Trend analysis of ALT in Case 5

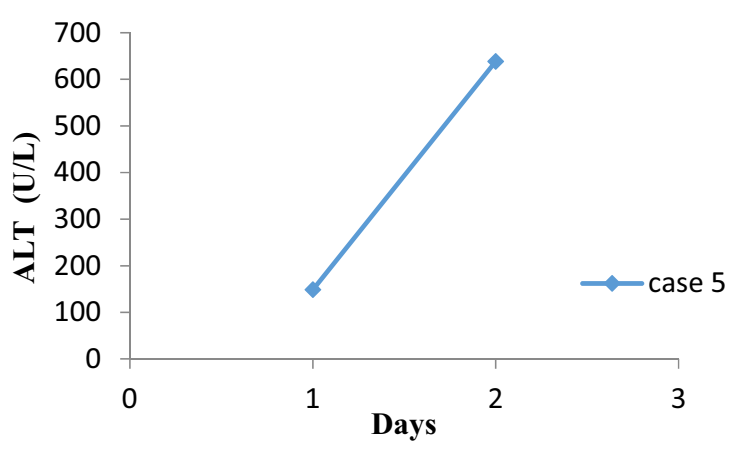

(c) Trend analysis of AST in Case 5

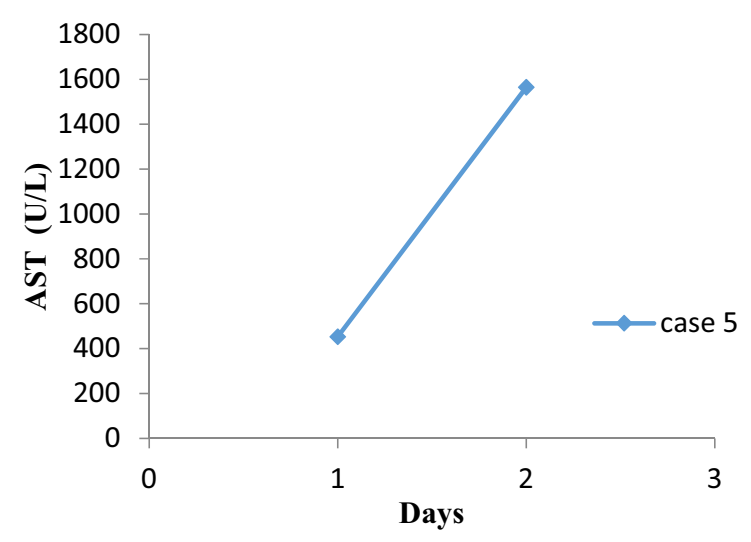

Fig. 5 Trend analysis of Biomarkers in Case 5. a Trend analysis of BUN in Case 5, b Trend analysis of ALT in Case 5, $\mathbf{c}$ Trend analysis of AST in Case 5

through droplets or direct contact, mild symptoms start developing in most of the patients. There are few asymptomatic patients. Few patients also show severe symptoms that include ARDS, multiple organ failure, pneumonia, or pulmonary edema. Due to the challenge of no specific treatment being available, it has become even more important to understand the disease and diagnose it at the earliest [6]. 
Table 3 Laboratory Abnormalities in COVID-19 progression [42]

\begin{tabular}{|c|c|c|}
\hline Parameter & Abnormalities & Clinical significance \\
\hline LDH & Elevated & Tissue damage, onset of sepsis \\
\hline ALT & Elevated & Liver damage \\
\hline AST & Elevated & Liver damage \\
\hline T. Billirubin & Elevated & Liver injury \\
\hline Creatinine & Elevated & Kidney injury \\
\hline D-Dimer & Elevated & Septic shock, coagulation activation \\
\hline PCT & Elevated & Pulmonary disease, sepsis, viral pneumonia, bacterial infection \\
\hline CRP & Elevated & Inflammation, infection, sepsis, viremia \\
\hline Ferritin & Elevated & Pro-inflammation, \\
\hline IL-6 & Elevated & Inflammation, cytokine strom \\
\hline Albumin & Decreased & Liver function \\
\hline Blood gas & Deranged & Critical care management \\
\hline High sensitive cardiac troponin I & Elevated & Cardiac injury \\
\hline NT proBNP/ BNP & Elevated & Cardiac injury \\
\hline Lactate & Elevated & Sepsis, tissue damage \\
\hline BUN & Elevated & Kidney injury \\
\hline Neutrophils & Elevated & Acute inflammation, bacterial infection \\
\hline Lymphocytes & Decrease & Viral, bacterial, fungal and parasitic infection \\
\hline Leucocytes & Elevated & Systemic inflammation and chronic disorder \\
\hline Platelet & Decrease & Viral infection \\
\hline
\end{tabular}

In this study, we have focused on the laboratory finding in COVID-19 patients with an aim, for a better understanding of the novel coronavirus disease. We have evaluated various biomarkers that include inflammatory markers, pulmonary, kidney, liver, cardiac, and tissue injury markers in this study. The biomarkers are correlated with hematological derangement of markers. One of the important biomarker considered in this study is Interleukin6 (IL-6). IL-6 is a pleotropic marker that plays an important role to integrate defense against infection [30], which is seen to elevate in COVID-19 patients. Studies have shown that IL-6 which is a pro-inflammatory marker plays an important role in patients with lung damage that is caused by SARS CoV-2 [31]. In response to an infection or tissue injury, IL-6 has a prompt and transient production. This production of IL-6 leads to the stimulation of an acute phase response that contributes to the host defense mechanism [32].

Another marker that shows elevation in COVID-19 patients includes D-dimer, which is the primary enzymatic degradation product of cross-linked fibrin by plasmin $[33,34]$. Studies have shown that the risk of the patient having a septic shock and sepsis increases with increasing levels of D-dimer [34].

Cardiac biomarkers like hs Troponin I and NT pro BNP which play a role in cardiac injury and myocardial infarction [34-37]. Also tend to show an increase. Studies have shown that in patients with COVID-19 disease, the prognostic effect of cardiac biomarkers (NT pro BNP and hs troponin I) could be directly or indirectly related to cardiac injury. Binding of SARS CoV2 with ACE2 leads to restricted synthesis of angiotensin 1-7 and also leads to elevated levels of angiotensin 2. Angiotensin 2 aids the secretion of NT pro BNP and cardiac Troponin whereas the anti-inflammatory effect is exerted by the elevated levels of angiotensin 1-7 [21].

Kidney injury marker and Liver injury markers like Creatinine, BUN, and AST, ALT is also seen to increase in COVID-19 [34]. Procalcitonin, a precursor of calcitonin, used as a biomarker in sepsis, viral pneumonia, and obstructive pulmonary disease [19] also tends to rise in COVID-19 patients.

An elevated level of ferritin plays a role in pro-inflammation [38]. The production of ferritin under inflammatory conditions of the body is an important acute phase reactant. These inflammatory conditions include infections as well as, hematologic, malignant, and rheumatologic conditions. Ferritin tends to reduce the iron supply as a part of a defense mechanism, hence incase of high pathogenic load elevated levels of ferritin are observed [39]. Along with the reduction in iron supply, ferritin also tends to regulate 
cytokine (responsible for cytokine proinflammatory storm) synthesis and release [40]

Another acute phase reactant synthesized in response to infection or inflammation is C-reactive protein (CRP). CRP is synthesized by the liver. A dramatic increase in serum concentration is seen in acute inflammation hence making CRP a more important marker for viremia and sepsis. CRP also plays a role in inflammatory cytokine activation hence contributing to the proinflammatory cycle [39].

Patients with severe sepsis also show an association with elevated levels of lactate dehydrogenase (LDH). LDH is known to depict the extent of tissue damage. Studies show when LDH levels do not normalize within $48 \mathrm{~h}$ of sepsis onset is related to the prediction of patient mortality [41].

Therefore, routine blood tests in the laboratory medicine department serve as a potential diagnostic tool for COVID19 [43]. Elevated biomarkers include CRP, ALT, AST, LDH, BUN, Creatinine, PCT, Lactate, Ferritin, IL6, etc. Biomarkers that tend to decrease with COVID-19 patients observed from our study include, PO2, SO2, Albumin, total protein. Along with the above markers, hematological markers also play an important role in the COVID-19 prognosis. Neutrophil and WBC Count show an increase in coronavirus infection and lymphocytes and eosinophils tend to show a decrease. The trend analysis of the various biomarkers in COVID-19 patients carried out in the study will help in understanding the course of COVID-19 treatment while chasing the cytokine storm in ARDS. Since the pathogenecity of the Novel Coronavirus is yet not clearly understood in spite of several ongoing studies [44], Routine laboratory testing might aid in symptom-dependent treatment regime till the complete understanding of the same.

We believe that the data considered for the study shows the importance of routine laboratory biomarkers in the coronavirus disease 2019 (COVID-19) and it will aid in a better understanding of the disease.

The study has a few limitations. The first being a smaller sample size and since it's retrospective data, not all the patients carried out all blood tests.

\section{Conclusions}

In summary, we can conclude from the study that COVID19 patients show significant abnormalities in the routine laboratory biomarkers characterized by an abnormality in AST, ALT, T Billirubin, Creatinine, CRP, PCT, LDH, IL6, Ferritin, Blood Gases and Albumin in COVID 19 patients.

A number of biomarkers that include IL-6, NT Pro BNP, hs Troponin I, PCT, CRP, Ferritin, LDH, Creatinine, BUN, AST, ALT, tend to increase in COVID-19 patients. Biomarkers like PO2, SO2, Total protein, Albumin, tend to decrease in such patients.

\section{Declarations}

Conflict of interest The author declares no conflict of interest.

Ethical Approval Institutional Ethics Committee Approval was taken.

\section{References}

1. Lippi G, Plebani M. Laboratory abnormalities in patients with COVID-2019 infection. Clin Chem Lab Med (CCLM). 2020;58(7):1131-4. https://doi.org/10.1515/cclm-2020-0198.

2. Zhou F, Ting Y, Ronghui D, Fan G, Liu Y, Liu Z, et al. Clinical course and risk factors for mortality of adult inpatients with COVID-19 in Wuhan, China: a retrospective cohort study. The Lancet. 2020;395(10229):1054-62. https://doi.org/10.1016/ S0140-6736(20)30566-3.

3. Guan WJ, Zheng-yi Ni YH, Liang W-H, Chun-quan O, He J-X, et al. Clinical characteristics of coronavirus disease 2019 in China. N Engl J Med. 2020;382:1708-20. https://doi.org/10. 1056/NEJMoa2002032.

4. Mitra P, Misra S, Sharma P. COVID-19 pandemic in India: what lies ahead. Ind J ClinBiochem. 2020;35:257-9. https://doi.org/10. 1007/s12291-020-00886-6.

5. World Health Organization Coronavirus (COVID-19) Dashboad, as of 2:35 PM CEST, 18th April, 2021. https://covid19.who.int.

6. Gao Y, Li T, Han M, Li X, Dong W, Yuanhong X, et al. Diagnostic utility of clinical laboratory data determinations for patients with the severe COVID-19. J Med Virol. 2020;92(7):791-6. https://doi.org/10.1002/jmv.25770.

7. Yong-JianGeng Z-YW, Qian H-Y, Ji Huang C, Lodato R, Castriotta RJ. Pathophysiological characteristics and therapeutic approaches for pulmonary injury and cardiovascular complications of coronavirus disease. Cardiovasc Pathol. 2019;2020(47):107228.

8. TanuSinghal A. Review of coronavirus disease-2019 (COVID19). Indian J Pediatr. 2020;87(4):281-6.

9. Lingeswaran M, Goyal T, Ghosh R, Suri S, Mitra P, Misra S, Sharma P. Inflammation, immunity and immunogenetics in COVID-19: a narrative review. Indian $J$ Clin Biochem. 2020;35(3):260-73. https://doi.org/10.1007/s12291-020-00897-3.

10. Lippi G, Simundic A-M, Plebani M. Potential preanalytical and analytical vulnerabilities in the laboratory diagnosis of coronavirus disease 2019 (COVID-19). Clin Chem Lab Med. 2020;58(7):1070-6.

11. AlqahtaniID JS, Oyelade T, Aldhahir AM, Alghamdi SM, Almehmadi M, Alqahtani AS, et al. Prevalence, severity and mortality associated with COPD and smoking in patients with COVID-19: a rapid systematic review and meta-analysis. PLoS One. 2020;15(5):e0233147. https://doi.org/10.1371/journal.pone. 0233147.

12. Tobacco use and COVID-19. 11 May 2020 Statement. Geneva: World Health Organization. https://www.who.int/news-room/ detail/11-05-2020-who-statement-tobacco-use-and-covid-19. Accessed: 14 May 2020

13. Huang C, Wang Y, Li X, LiliRen JZ, Yi H, et al. Clinical features of patients infected with 2019 novel coronavirus in Wuhan, China. Lancet. 2020. https://doi.org/10.1016/S01406736(20)30183-5.

14. Götzinger F, Santiago-García B, AntoniNoguera-Julián ML, Lancella L, Calò FI, Carducci, , et al. COVID-19 in children and adolescents in Europe: a multinational, multicentre cohort study. Lancet Child Adolesc Health. 2020. https://doi.org/10.1016/ S2352-4642(20)30177-2. 
15. Riphagen S, Gomez X, Gonzalez-Martinez C, Wilkinson N. ParaskeviTheocharis. Hyperinflammatory shock in children during COVID-19 pandemic. Lancet. 2020;395(10237):1607-8.

16. DeBiasi RL, Song X, Delaney M, Bell M, Smith K, Pershad J, et al. Severe coronavirus disease-2019 in children and young adults in the Washington, DC metropolitan region. J Pediatr. 2020. https://doi.org/10.1016/j.jpeds.2020.05.007.

17. Jones VG, Mills M, Suarez D, Hogan CA, Debra Yeh J, Segal B, et al. COVID-19 and Kawasaki disease: novel virus and novel case. Hosp Pediatr. 2020. https://doi.org/10.1542/hpeds.20200123.

18. Plebani M, Laposata M, Lippi G. A manifesto for the future of laboratory medicine professionals. Clin Chim Acta. 2019;489:49-52.

19. Samsudin I, Vasikaran SD. Clinical utility and measurement of procalcitonin. Clin Biochem Rev. 2017;38(2):59-68.

20. Abbaspour N, Hurrell R, Kelishadi R. Review on iron and its importance for human health. J Res Med Sci. 2014;9:164-74.

21. COVID-19 Coronavirus Real-Time PCR Kit. https://www.who. int/diagnostics_laboratory/eual/eul_0515_202_00_covid19_cor onavirus_real_time_pcr_kit_ifu.pdf?ua $=1$. Assessed 15th August 2020

22. Hoffmann M, Kleine-Weber H, Krüger N, Müller M, Drosten C, Pöhlmann S. The novel coronavirus 2019 (2019-nCoV) uses the SARS-coronavirus receptor ACE2 and the cellular protease TMPRSS2 for entry into target cells. bioRxiv. 2020. https://doi. org/10.1101/2020.01.31.929042.

23. Letko M, Marzi A, Munster Vincent. Functional assessment of cell entry and receptor usage for SARS-CoV-2 and other lineage B betacoronaviruses. Nat Microbiol. 2020. https://doi.org/10. 1038/s41564-020-0688-y.

24. Zhang H, Kang Z, Gong H, Da X, Wang J, Li Z, Cui X, Xiao J, et al. The digestive system is a potential route of 2019-nCov infection: a bioinformatics analysis based on single-cell transcriptomes. bioRxiv. 2020. https://doi.org/10.1101/2020.01.30. 927806.

25. Zhu N, Zhang D, Wang W, Li X, Yang Bo, Song J, et al. A Novel Coronavirus from Patients with Pneumonia in China, 2019. N Engl J Med. 2020;382(8):727-33.

26. Liu Z, Bing X, Zhi XZ. An update on the epidemiological characteristics of novel coronavirus pneumonia (COVID-19). Chin J Epidemiol. 2020. https://doi.org/10.3760/cma.j.issn.02546450.2020.02.003.

27. Henry BM, Santos MH, de Oliveira S, Benoit MP, Lippi G. Hematologic biochemical and immune biomarker abnormalities associated with severe illness and mortality in coronavirus disease 2019 (COVID-19): a meta-analysis. Clin Chem Lab Med. 2020. https://doi.org/10.1515/cclm-2020-0369.

28. Lippi G, Plebani M. The critical role of laboratory medicine during coronavirus disease 2019 (COVID-19) and other viral outbreaks. Clin Chem Lab Med. 2020. https://doi.org/10.1515/ cclm-2020-0240.

29. Joost Wiersinga W, Rhode A, Cheng AC, Peacock SJ, Prescott HC. Pathophysiology transmission diagnosis and treatment of coronavirus disease 2019 (COVID-19). JAMA. 2020. https://doi. org/10.1001/jama.2020.12839.
30. Rose-John S, Winthrop K, Calabrese L. The role of IL-6 in host defence against infections: immunobiology and clinical implications. Nat Rev Rheumatol. 2017. https://doi.org/10.1038/ nrrheum.2017.83.

31. Grifoni E, Valoriani A, Cei F, Lamanna R, Gelli AMG, Ciambotti B, Vannucchi V, Moroni F, et al. Interleukin- 6 as prognosticator in patients with COVID-19. J Infect. 2020;81(3):452-82.

32. Tanaka T, Narazaki M, Kishimoto T. IL-6 in inflammation, immunity, and disease. Cold Spring Harb Perspect Biol. 2014;6(10):a016295.

33. Sathe PM, Patwa UD. D Dimer in acute care. Int J Crit Illn Inj Sci. 2014;2014:229-32.

34. Kavsak PA, de Wit K, Worster A. Emerging key laboratory tests for patients with COVID-19. Clin Biochem. 2020. https://doi.org/ 10.1016/j.clinbiochem.2020.04.009.

35. Bhutta ZA, Basnyat B, Saha S, Laxminarayan R. COVID-19 risks and response in South Asia. BMJ. 2020. https://doi.org/10.1136/ bmj.m1190.

36. Bularga A, Lee KK, Stewart S, Ferry AV, Chapman AR, Marshall L, et al. High-sensitivity troponin and the application of risk stratification thresholds in patients with suspected acute coronary syndrome. Circulation. 2019. https://doi.org/10.1161/CIRCULA TIONAHA.119.042866.

37. Gao L, Jiang D, Wen X-S, Cheng X-C, Sun M, He B, You L-N, et al. Prognostic value of NT-proBNP in patients with severe COVID-19. Resp Res. 2020. https://doi.org/10.1186/s12931-02001352-w.

38. Abbaspour N, Hurrell R, Kelishadi R. Review on iron and its importance for human health. J Res Med Sci. 2014;19:164-74.

39. Asghar MS, HaiderKazmi SJ, Khan NA, Akram M, Hassan M, Rasheed U, Ahmed Khan S. Poor prognostic biochemical markers predicting fatalities caused by COVID-19: a retrospective observational study from a developing country. Cureus. 2020;12(8):e9575. https://doi.org/10.7759/cureus.957527a5.

40. Giemza-Stokłosa J, Islam MA, Kotyla PJ. Hyperferritinaemia: an iron sword of autoimmunity. Curr Pharm Des. 2019;25:2909-18. https://doi.org/10.2174/1381612825666190709202804.

41. Zein JG, Lee GL, Tawk M, Dabaja M, Kinasewitz GT. Prognostic significance of elevated serum lactate dehydrogenase (LDH) in patients with severe sepsis. Chest. 2004;126:873. https://doi.org/10.1378/chest.126.4_MeetingAbstracts.873S.

42. IFCC Information Guide on COVID-19 (2020). https://www.ifcc. org/ifcc-news/2020-03-26-ifcc-information-guide-on-covid-19. Accessed on 30th November 2020

43. Ferrari D, Motta A, Strollo M, Banfi G, Locatelli M. Routine blood tests as a potential diagnostic tool for COVID-19. Clin Chem Lab Med. 2020. https://doi.org/10.1515/cclm-2020-0398.

44. Pal A, Squitti R, Picozza M, Pawar A, Rongioletti M, Dutta AK, Sahoo S, Goswami K, Sharma P, Prasad R. Zinc and COVID-19: basis of current clinical trials. Biol Trace Elem Res. 2020;22:1-11. https://doi.org/10.1007/s12011-020-02437-9.

Publisher's Note Springer Nature remains neutral with regard to jurisdictional claims in published maps and institutional affiliations. 